\title{
Desenvolvimento da Escala de Reabilitação Psicossocial para Usuários de
}

\section{Substâncias Psicoativas}

\author{
Development of the Psychosocial Rehabilitation Scale for Users of Psychoactive Substances \\ Desarrollo de la Escala de Rehabilitación Psicosocial para Usuarios de Sustancias Psicoactivas
}

Recebido: 23/05/2021 | Revisado: 30/05/2021 | Aceito: 02/06/2021 | Publicado: 18/06/2021

Paula Hayasi Pinho

ORCID: https://orcid.org/0000-0001-8922-0699 Universidade Federal do Recôncavo da Bahia, Brasil E-mail: paulahpinho@gmail.com

Heloísa Claro Garcia

ORCID: https://orcid.org/0000-0003-1504-7074 Universidade Estadual de Campinas, Brasil E-mail: clarohg@unicamp.br

Gabriella de Andrade Boska

ORCID: https://orcid.org/0000-0002-5827-6486 Universidade de São Paulo, Brasil E-mail: gabriellaboska@usp.br

Márcia Aparecida Ferreira de Oliveira

ORCID: https://orcid.org/0000-0002-1069-8700 Universidade de São Paulo, Brasil E-mail: marciaap@usp.br

\begin{abstract}
Resumo
Pessoas que fazem uso de substâncias psicoativas com frequência tem seus direitos eximidos. A Reabilitação Psicossocial é a diretriz de cuidado do modelo psicossocial e busca integrar o indivíduo à sua rede e a um contexto social mais amplo. Objetivo: Construir uma escala de Reabilitação Psicossocial para usuários de substâncias psicoativas e calcular a sua validade de conteúdo. Métodos: Estudo metodológico, de construção de itens de uma escala após revisão integrativa. A escala foi submetida à avaliação de 5 juízes especialistas. Resultados: 35 artigos subsidiaram a criação de 33 itens da escala. Os itens dividiram-se nos domínios da Reabilitação Psicossocial moradia, trabalho e renda, rede social, autonomia e cidadania, e cuidado em comunidade. As respostas aos itens são de tipo Likert, 1 a 5. O Índice de Validade de Conteúdo da escala foi de 0,85 para pertinência dos itens e 0,847 para adequabilidade à população alvo. A escala é considerada válida para testes com a população de estudo em sua versão final de 32 itens. Considerações Finais: A escala proposta é inédita e possui conteúdo válido para a área de álcool e outras drogas. Contribuirá para a avaliação e o planejamento do cuidado aos usuários dos serviços comunitários de saúde mental, na perspectiva da Reabilitação Psicossocial.
\end{abstract}

Palavras-chave: Psicometria; Álcool e outras drogas; Estudos de validação; Reabilitação psicossocial.

\begin{abstract}
People who use psychoactive substances often have their rights exempted. Psychosocial Rehabilitation is the guideline for the care of the psychosocial model and seeks to integrate the individual into their network and into a broader social context. Objective: To build a Psychosocial Rehabilitation scale for users of psychoactive substances and calculate their content validity. Methods: Methodological study, of constructing items of a scale after integrative review. The scale was submitted to the evaluation of 5 expert judges. Results: 35 articles supported the creation of 33 items of the scale. The items were divided into the domains of Psychosocial Rehabilitation housing, work and income, social network, autonomy and citizenship, and community care. The responses to items are Likert type, 1 to 5 . The Content Validity Index of the scale was 0.85 for the pertinence of the items and 0.847 for suitability to the target population. The scale is considered valid for tests with the study population in its final version of 32 items. Final Considerations: The proposed scale is unprecedented and has valid content for the area of alcohol and other drugs. It will contribute to the evaluation and planning of care for users of community mental health services, from the perspective of Psychosocial Rehabilitation.
\end{abstract}

Keywords: Psychometrics; Alcohol and other drugs; Validation studies; Psychosocial rehabilitation.

\section{Resumen}

Las personas que consumen sustancias psicoactivas se les renuncia con frecuencia a sus derechos. La Rehabilitación Psicosocial es la pauta asistencial del modelo psicosocial y busca integrar al individuo en su red y en un contexto social más amplio. Objetivo: Construir una escala de Rehabilitación Psicosocial para usuarios de sustancias 
psicoactivas y calcular su validez de contenido. Métodos: Estudio metodológico, de construcción de ítems en una escala luego de una revisión integradora. La escala fue sometida a la evaluación de 5 jueces expertos. Resultados: 35 artículos apoyaron la creación de 33 ítems en la escala. Los ítems se dividieron en las áreas de rehabilitación psicosocial vivienda, trabajo e ingresos, red social, autonomía y ciudadanía y atención comunitaria. Las respuestas a los ítems son de tipo Likert, de 1 a 5 . El Índice de Validez de Contenido de la escala fue 0,85 para la pertinencia de los ítems y 0,847 para la adecuación a la población objetivo. La escala se considera válida para pruebas con la población de estudio en su versión final de 32 ítems. Consideraciones finales: La escala propuesta no tiene precedentes y tiene contenido válido para el área de alcohol y otras drogas. Contribuirá a la evaluación y planificación de la atención a los usuarios de los servicios comunitarios de salud mental, desde la perspectiva de la Rehabilitación Psicosocial.

Palabras clave: Psicometría; Alcohol y otras drogas; Estudios de validación; Rehabilitación psicosocial.

\section{Introdução}

A Política Nacional de Saúde Mental, Álcool e Outras Drogas é norteada pelas diretrizes da Reforma Psiquiátrica brasileira e da Lei ${ }^{\circ} 10.216$ (2001), que reorienta o modelo assistencial superando a característica asilar/manicomial e garante os direitos de cidadania da pessoa com transtornos mentais e com transtornos decorrentes do uso de Substâncias Psicoativas (SPA).

Como principal diretriz desse modelo assistencial temos a Reabilitação Psicossocial (RP). Esta é definida pelo Ministério da Saúde (2004) como um conjunto de ações e/ou práticas que visam o protagonismo do indivíduo, de iniciativas integradas ao território, de trabalho/economia solidária, habitação, educação, cultura e saúde, buscando a emancipação desses indivíduos e de seus familiares, garantindo seus direitos de cidadania e contratualidade no território. Em síntese, busca a reconstrução do sujeito em três grandes cenários: o habitat, a rede social e o trabalho com valor social e com isso, viabiliza o aumento da capacidade do sujeito de exercer sua cidadania em todo o seu contexto de vida (Saraceno, 1999).

Para além disso, a RP é uma estratégia e uma vontade política de cuidado aos indivíduos em situação de vulnerabilidade, visando devolver sua autonomia e capacidade de escolha, sendo um agente facilitador no processo de trocas sociais, minimizando eventuais barreiras e restituindo, portanto, direitos, cidadania plena e papéis sociais (Pinho, Oliveira, Claro, Pereira, e Almeida, 2013). Os autores ressaltam, ainda, a importância da discussão do significado do tratamento em si e dos recursos (humanos, comunitários e sociais) que corroboram com a estratégia, uma vez que a literatura no campo de RP é escassa e, muitas vezes, não relacionada (na literatura internacional) com a prevenção e tratamento do consumo de SPA (Pinho et al., 2013).

Pessoas que fazem uso de substâncias psicoativas (SPA) com frequência tem seus direitos eximidos, não tendo acesso a moradia, rede social, trabalho e renda, autonomia e cidadania, beneficiando-se de estratégias neste campo de atenção, para ser produzido um cuidado integral e reabilitador (United Nations Office on Drugs and Crime [UNODC], 2014). A RP desperta a necessidade do reconhecimento do usuário como um cidadão de direitos em um ambiente que deve buscar favorecer a sua autonomia e a participação democrática. Os Centros de Atenção Psicossocial (CAPS), neste caso sobretudo os voltados para álcool e outras drogas (CAPS AD), são os lugares de cuidado para esta população que possibilitam desenvolver estratégias claras voltadas para a RP (Ministério da Saúde, 2002).

A RP pode ser então entendida, como um processo que determina o aumento da capacidade da pessoa de utilizar oportunidades e recursos pessoais e coletivos, num percurso de inclusão social por meio do crescimento de sua contratualidade psicológica e social e da constituição material do direito à cidadania. Portanto, atenção deve ser dada aos indicadores de resultados sociais/ interpessoais relacionados a RP, distintos do desfecho clínico (Saraceno, 1999).

Na contramão da exclusão e estigmatização dos usuários de SPA, a RP busca a garantia de seus direitos humanos, uma vez que estão inseridas no mundo e que este é apenas um para todos. Ainda, recupera o poder de contratualidade, de trocas e de negociações. Supera o modelo biomédico psiquiátrico tradicional, convoca não só os usuários, mas também os 
familiares, os profissionais de saúde, os gestores das políticas públicas e a comunidade para potencializar os esforços de cuidado em RP bem como todos os recursos: afetivos (relações pessoais, familiares, amigos, etc.), sanitários (serviços de saúde), sociais (moradia, trabalho, educação, etc.), econômicos (dinheiro, previdência etc.), culturais, religiosos e de lazer.

Estudos de validação de escalas e instrumentos são realizados para fornecer ferramentas de diagnóstico, planejamento de tratamento e avaliação da evolução do indivíduo. Com a utilização destes instrumentos, torna-se possível apreender o sentido que alguns acontecimentos têm para os indivíduos, assim como os fatores que os predispõem, as relações existentes entre as causas e as consequências e a efetividade dos tratamentos (Ferrari, 2009). Diversos instrumentos e escalas foram adaptados à cultura brasileira, nas últimas décadas, principalmente os que tratam sobre os problemas de saúde mais prevalentes, gerados pelas condições de vida dos indivíduos, com o objetivo de realizar a avaliação objetiva das pessoas e de sua evolução ao longo do tratamento (Ferrari, 2009).

Não encontramos na literatura internacional instrumentos que mensuram as necessidades e evolução clínica nos três eixos da RP que nos permitam planejar e avaliar o tratamento do usuário de SPA. Entendemos que mensurar a RP é um indicador de resultado para avaliar os serviços no cuidado em álcool e outras drogas, planejar o cuidado e avaliar a evolução clínica dos indivíduos.

Neste sentido, este estudo tem por objetivo construir uma Escala de Reabilitação Psicossocial para Usuários de SPA com base em uma revisão integrativa da literatura e calcular a sua validade de conteúdo.

\section{Metodologia e Análise dos Dados}

Trata-se de um estudo metodológico de construção de itens da escala, que mensura a RP do indivíduo usuário de SPA (Polit, Beck, \& Hungler, 1995). Os itens que compõe a escala foram desenvolvidos por meio de uma revisão integrativa da literatura em cinco etapas: 1) definição da pergunta de pesquisa; 2) seleção da amostra (após definição dos critérios de inclusão e exclusão); 3) caracterização dos estudos (definição dos dados a serem coletados de acordo com um instrumento); 4) análise dos resultados; e 5) apresentação e discussão dos resultados (Soares et al., 2014).

Este tipo de estudo possui aspectos quantitativos, quando consideramos a análise da volumetria das variáveis encontradas na literatura para a construção da escala, índice de validade de conteúdo calculado com base na opinião dos especialistas, entre outros. Entretanto, possui também aspectos qualitativos para a análise das respostas dos juízes e síntese das sugestões em itens para a mensuração da RP. Este esforço é necessário para garantir itens que diferenciem e quantifiquem, em um espectro de gravidade, os indivíduos quanto às suas urgências relacionadas à RP com itens de qualidade (Claro et al., 2011).

A coleta de dados aconteceu em julho de 2020, nas bases de dados Biblioteca Virtual em Saúde -BVS (Lilacs), portal PubMed que engloba o Medline e duas bibliotecas digitais Scielo e PsycINFO. Para o levantamento dos estudos utilizamos os seguintes descritores e suas combinações nas línguas portuguesa e inglesa: "Reabilitação Psicossocial" AND "Álcool” AND "Drogas". Os critérios de inclusão são artigos publicados em português, inglês e espanhol, sem corte temporal.

A busca com os termos acima descritos resultou em uma amostra de artigos que embasaram os itens da escala. $\mathrm{O}$ objetivo foi verificar os temas e características da RP descritos na literatura e formular itens que possibilitem mensurar as necessidades do indivíduo nos três eixos, além disso, avaliamos também questões como autonomia e cidadania, e cuidado em comunidade, intrinsecamente ligados à RP.

Para a extração dos dados, utilizamos de formulário eletrônico para estruturar e sistematizar a busca dos artigos e realizar a categorização dentro dos temas pertinentes à RP. Para cada artigo, coletamos informações sobre: base de dados da busca; título; autores; ano; país de publicação; idioma; aborda SPA como tema central? aborda os eixos da RP renda/trabalho, 
moradia/habitar e rede social? aborda o cuidado em comunidade como estratégia de reabilitação? e campo para observação para outras informações pertinentes.

\section{Avaliação da Escala por Juízes Especialistas e Validade de Conteúdo}

A coleta de dados com os juízes foi feita entre julho e agosto de 2020. Os juízes foram informados dos objetivos da pesquisa, orientados em como proceder ao preenchimento do formulário e receberam uma cópia assinada do Termo de Consentimento Livre e Esclarecido (TCLE), aprovado pelas instâncias éticas competentes. Após ciência e registro do TCLE preencheram os formulários e os dados foram armazenados em servidor seguro da universidade, em planilha com acesso protegido.

As respostas dos juízes foram coletadas por meio de formulário eletrônico enviado via e-mail. Foram apresentados a eles os 33 itens da escala, e eles foram convidados a avaliar cada item quanto à sua pertinência ( $\mathrm{O}$ item identifica questões pertinentes à reabilitação psicossocial do indivíduo?) e adequabilidade quanto à compreensão pelo público alvo (Está adequado quanto à facilidade de compreensão pelo público alvo?). Os juízes possuíram as seguintes possibilidades de respostas: "adequado"; "adequado, mas, precisa revisão"; "inadequado, remover".

Os juízes especialistas foram selecionados de acordo com os seguintes critérios: Doutor na área de saúde mental ou uso de substâncias psicoativas, que possuíam familiaridade com o uso de escalas no contexto da clínica em saúde mental e uso de álcool e outras drogas e com experiência na área de pesquisa. Foram convidados cinco juízes, que avaliaram os itens da escala em sua totalidade, desta forma, todos foram incluídos.

Mensuramos o Índice de Validade de Conteúdo - IVC, (Content Validity Index) que avalia a resposta dos juízes quanto à representatividade de cada item da medida proposta em relação ao conteúdo (constructo) estudado. Em nosso caso, cada um dos 33 itens foi avaliado pelos juízes quanto à sua adequação ao constructo da Reabilitação Psicossocial de usuários de SPA. A escala é considerada válida se obtiver um IVC de 0,80 (Polit et al. 1995; Waltz \& Bausell, 1981).

Calculamos o IVC utilizando o SPSS 20. Utilizamos a média da razão do número de itens avaliados como adequados por ambos os juízes em cada um dos pares de juízes possível (a), dividida pelo total de itens da escala (b), onde IVC médio = a/b (Liposck, Rosa, \& Savall, 2007; Waltz \& Bausell, 1981; Waltz, Strickland, \& Lenz, 1991).

Este estudo obedeceu aos princípios éticos da Resolução no 466/12, do Conselho Nacional de Saúde, dada a natureza da coleta de dados com os juízes que avaliaram a escala e foi aprovado junto ao Comitê de Ética em Pesquisa (CEP) da Escola de Enfermagem da Universidade de São Paulo, sob o Parecer N ${ }^{\circ}$ 3.519.949/19 e pelo CEP da Secretaria Municipal de Saúde do Município de São Paulo, sob o Parecer No 3.615.072/19.

\section{Resultados}

Encontramos 18 artigos na PubMed, 17 na Scielo, 25 na Psycinfo e 19 na BVS, totalizando 79 artigos. Após eliminação dos 26 registros duplicados, analisamos, na íntegra, 53 artigos. Destes, 20 foram excluídos, pelos seguintes motivos: não abordavam RP seus eixos ou cuidado em comunidade (14), não abordavam tratamento para usuários de álcool e outras drogas (1), estudos experimentais com animais (1) ou por tratar-se de resenha de livros (4). Assim, a amostra final foi composta por 33 artigos.

Dos 33 artigos selecionados, 1 foi publicado em idioma espanhol, 22 em inglês, 10 em português. Apenas 1 dos artigos foi publicado em 1978, 7 foram publicados entre 1985 e 1999, 15 artigos entre 2000 e 2014, e 10 artigos publicados nos últimos 5 anos. Após proceder à leitura integral e categorização dos artigos, identificamos, por meio de leitura crítica, os temas listados na Tabela 1. 
Tabela 1: Temas abordados pelos artigos.

\begin{tabular}{|c|c|c|c|}
\hline & Variável & $\begin{array}{c}\text { Artigos } \\
(\mathbf{n}=33)\end{array}$ & $\begin{array}{c}\text { Itens } \\
(\mathbf{n}=\mathbf{3 3})\end{array}$ \\
\hline \multirow{2}{*}{$\begin{array}{c}\text { Temas dos } \\
\text { Artigos }\end{array}$} & $\begin{array}{l}\text { AOD TEMA CENTRAL (Alvarez et al., 2012; Barbosa et al., } \\
\text { 2015; Bergman \& Harris, 1985; Blondell et al., 2011; Bohn, 1993; } \\
\text { Braucht et al., 1978; George \& Ritz, 1993; Lefio et al., 2013; } \\
\text { Maisto et al., 2002; McKay et al., 2015; Pinho et al., 2008, 2009, } \\
\text { 2013; Secades-Villa et al., 2011; Sena et al., 2017; Sheidow et al., } \\
\text { 2012; Wandekoken et al., 2015; White \& White, 1989) }\end{array}$ & $\begin{array}{c}22 \\
(67 \%)\end{array}$ & $\begin{array}{l}\text { Não se } \\
\text { aplica }\end{array}$ \\
\hline & $\begin{array}{l}\text { AOD TEMA SECUNDÁRIO (Bellack \& Mueser, 1993; Brito et } \\
\text { al., 2012; Dell'Osso et al., 2018; Fals-Stewart \& O'Farrell, 2003; } \\
\text { Hyde \& Goldman, 1993; Judd et al., 2003; Lazar et al., 2016; } \\
\text { Lucchese et al., 2017; McCoy et al., 2003; Otto-Salaj et al., 1998; } \\
\text { Sordi et al., 2015; Stein et al., 2015) }\end{array}$ & $\begin{array}{c}13 \\
(39 \%)\end{array}$ & $\begin{array}{l}\text { Não se } \\
\text { aplica }\end{array}$ \\
\hline \multirow{5}{*}{$\begin{array}{l}\text { Categorias de } \\
\text { Divisão das } \\
\text { Informações } \\
\text { dos Artigos }\end{array}$} & $\begin{array}{l}\text { RENDA/TRABALHO (Barbosa et al., 2015; Judd et al., 2003; } \\
\text { Lazar et al., 2016; Maisto et al., 2002; McCoy et al., 2003; Pinho et } \\
\text { al., 2009, 2013; Sena et al., 2017; Sheidow et al., 2012) }\end{array}$ & $\begin{array}{c}16 \\
(48 \%)\end{array}$ & $5(15 \%)$ \\
\hline & $\begin{array}{l}\text { MORADIA (Braucht et al., 1978; Judd et al., 2003; Maisto et al., } \\
\text { 2002; McCoy et al., 2003; Pinho et al., 2009, 2013; Sena et al., } \\
\text { 2017) }\end{array}$ & $\begin{array}{c}14 \\
(42 \%)\end{array}$ & $6(18 \%)$ \\
\hline & $\begin{array}{l}\text { REDE SOCIAL (Alvarez et al., 2012; Barbosa et al., 2015; Bellack } \\
\text { \& Mueser, 1993; Bergman \& Harris, 1985; Braucht et al., 1978; } \\
\text { Brito et al., 2012; Fals-Stewart \& O'Farrell, 2003; Hyde \& } \\
\text { Goldman, 1993; Judd et al., 2003; Lefio et al., 2013; Maisto et al., } \\
\text { 2002; Pinho et al., 2008, 2009, 2013; Secades-Villa et al., 2011; } \\
\text { Sena et al., 2017; Sheidow et al., 2012; Wandekoken et al., 2015) }\end{array}$ & $\begin{array}{c}26 \\
(79 \%)\end{array}$ & $10(30 \%)$ \\
\hline & $\begin{array}{l}\text { AUTONOMIA E CIDADANIA (Alvarez et al., 2012; Barbosa et } \\
\text { al., 2015; Bergman \& Harris, 1985; Davison, 2000; Green et al., } \\
\text { 2012; Hyde \& Goldman, 1993; Lazar et al., 2016; Lefio et al., } \\
\text { 2013; Maisto et al., 2002; McKay et al., 2015; Pinho et al., 2008, } \\
\text { 2009, 2013; Secades-Villa et al., 2011; Sena et al., 2017; Sheidow } \\
\text { et al., 2012; Sordi et al., 2015; Stein et al., 2015; Wandekoken et } \\
\text { al., 2015) }\end{array}$ & $\begin{array}{c}12 \\
(36 \%)\end{array}$ & $4(12 \%)$ \\
\hline & $\begin{array}{l}\text { CUIDADO EM COMUNIDADE (Alvarez et al., 2012; Barbosa et } \\
\text { al., 2015; Bellack \& Mueser, 1993; Bergman \& Harris, 1985; Brito } \\
\text { et al., 2012; Davison, 2000; Dell'Osso et al., 2018; Green et al., } \\
\text { 2012; Judd et al., 2003; Junqueira-Ayres et al., 2017; Lefio et al., } \\
\text { 2013; Lucchese et al., 2017; Maisto et al., 2002; Otto-Salaj et al., } \\
\text { 1998; Sena et al., 2017; Stein et al., 2015; Wandekoken et al., } \\
\text { 2015) }\end{array}$ & $\begin{array}{c}24 \\
(72 \%)\end{array}$ & $8(24 \%)$ \\
\hline
\end{tabular}

Fonte: Autoras.

Procedemos, então à interpretação e síntese dos resultados. Ao analisar os conteúdos extraídos de cada artigo sobre o conceito de RP, sua importância no cuidado e desfechos desejados do cuidado, dividimos as contribuições dos artigos em cinco categorias: moradia, trabalho/renda, rede social, autonomia/cidadania, e cuidado em comunidade. A reinserção social, termo que também foi frequente nos artigos estudados, consideramos, em nossa análise, como algo que compreende todos estes cinco domínios, por considerarmos que não há inserção social sem cada um deles. 
Os 33 itens que elaboramos para a escala dividem-se em cinco domínios, compatíveis com as categorias apresentadas na Tabela 1. Apresentamos a seguir a lógica de cada um dos domínios dos itens da escala

\section{Renda/Trabalho}

Os artigos que abordaram esse tema expuseram, principalmente, que o consumo de SPA prejudica a situação financeira dos usuários. Muitos referem não possuir renda suficiente para necessidades básicas, e, ao iniciar ou agravar o uso de SPA, o absenteísmo, prejuízos psicossociais, entre outras questões relacionadas ao uso de drogas pioram ainda mais esta situação (Pinho et al., 2013; Pinho et al., 2009; Shidow, Mccart, Zajac, \& Davis, 2012; Maisto, Martin, Pollock, Cornelius, \& Chung, 2002; Sena et al., 2017; Barbosa et al., 2015; Judd, Thomas, Schwartz, Outcalt, \& Hough, 2003; Lazar et al., 2016; Mccoy et al., 2003).

Os estudos apontam ainda que prejuízos laborais são um dos indicativos de que o indivíduo precisa de ajuda relacionada a abuso ou dependência de SPA. Desta forma, uma escala que busque mensurar a reabilitação psicossocial dos usuários de SPA deve avaliar perdas provocadas ou exacerbadas, na visão do usuário, pelo uso presente. Considerando estes aspectos, elaboramos 5 itens relativos a este domínio.

\section{Moradia}

A situação de vulnerabilidade social dos indivíduos é máxima quando não possuem moradia. Quando há prejuízo ao trabalho e renda, a moradia é consequentemente prejudicada. Mais do que um lugar para morar, o indivíduo precisa pertencer, habitar. Propomos como importantes de serem avaliadas, neste domínio, questões relacionadas à percepção do usuário de que o uso de SPA lhe prejudicou em sua moradia. Além disso, para além do uso de SPA, é necessário avaliar se o usuário se sente confortável seguro, com liberdade ou satisfeito com sua situação atual de moradia (Pinho et al., 2013; Pinho et al., 2009; Maisto et al., 2002; Braucht, Kirby, \& Berry, 1978; Sena et al., 2017; Judd et al., 2003; Mccoy et al., 2003).

Dentro deste contexto, elaboramos 6 itens dentro deste domínio para a escala proposta.

\section{Rede Social}

Os artigos apontam que as relações, rede de apoio, rede social, rede de interação ou mapa de relações é essencial para que o usuário de SPA atinja bons resultados em seu tratamento para uso destas.

Como fortalecimento, fonte de apoio e até mesmo parte do tratamento, sobretudo nos serviços comunitários, percebemos a importância de se avaliar questões relacionadas a perdas ou problemas relacionados à rede social do indivíduo causadas pelo uso de SPA, bem como a qualidade, potência e satisfação do indivíduo quanto às suas interações familiares e com os amigos (Pinho et al., 2013; Wandekoken, Quintanilha, \& Dalbello, 2015; Lefio, Villarroel, Rebolledo, Zamorano, \& Rivas, 2013; Alvarez, Gomes, Oliveira, \& Xavier, 2012; Pinho et al., 2009; Pinho, Oliveira, \& Almeida, 2008; Sheidow et al., 2012; Maisto et al., 2002; Secades-Villa et al., 2011; Berhman \& Harris, 1985; Braucht et al., 1978; Sena et al., 2017; Barbosa et al., 2015; Judd et al., 2003; Fals-Stewart e O-Parrell, 2003; Hyde \& Goldman, 1993; Brito, Brito, Silva, \& Azevedo, 2012; Belack \& Mueser, 1993).

Desta forma, elaboramos 10 itens para a escala que avaliam estas questões, dentro do domínio rede social.

\section{Autonomia/Cidadania}

Levantamos nos artigos selecionados preocupações específicas com o grau no qual o indivíduo é capaz de cuidar de si mesmo, de conquistar o que precisa e exercer seus direitos na comunidade. 
Dentro desta questão, elaboramos 4 itens que avaliam a capacidade autopercebida do quanto o indivíduo toma as suas próprias decisões, do quanto pode cuidar de si mesmo, se sente-se pertencente aos ambientes que frequenta ou se limita-se por não sentir pertencente, e se sente que o uso de SPA lhe atrapalha em suas funções, papéis e desejos (Pinho et al., 2013; Wandekoken et al., 2015; Lefio et al., 2013; Alvarez et al., 2012; Pinho et al., 2009; Pinho et al., 2008; Sheidow et al., 2012; Maisto et al., 2002; Secades-Villa et al., 2011; Berhman \& Harris, 1985; Sena et al., 2017; Mckay et al., 2015; Barbosa et al., 2015; Lazar et al., 2016; Hyde \& Goldman, 1993; Sordi, Sordi, Bigatto, Santos, \& Machado, 2015; Stein et al., 2015; Davison, 2000; Green, Wisdom, Wolfe, \& Firemark, 2012).

\section{Cuidado em Comunidade}

Os artigos nos permitem avaliar que não há possibilidade de RP fora da comunidade. Indivíduos excluídos, asilados em manicômios, não possuem autonomia, seus direitos são cerceados, não havendo possibilidade de exercer nenhuma das questões levantadas como importantes anteriormente. Desta forma, entendemos ser importante avaliar se, quando há necessidade, o indivíduo em crise, abstinência ou intoxicação é encaminhado para um hospital geral (e não a um hospital psiquiátrico), se é acompanhado por uma equipe de atenção primária à saúde, se é acompanhado por um serviço comunitário, e se está satisfeito e vê resultados deste cuidado em rede. Desta forma, elaboramos 8 itens que buscam avaliar esses aspectos da RP na vida dos indivíduos (Wanderkoken et al., 2015; Lefio et al., 2013; Alvarez et al., 2012; Maisto et al., 2002; Berhman \& Harris, 1985; Sena et al., 2017; Barbosa et al., 2015; Judd et al., 2003; Dell'Osso et al., 2018; Lucchese et al., 2017; Brito et al., 2012; Stein et al., 2015; Otto-Salag, Kelly, \& Stevenson, 1998; Bellack \& Mueser, 1993).

\section{Versão da Escala para Avaliação}

Propomos aqui, portanto, uma escala que compreende 4 itens no domínio autonomia/cidadania, 6 itens no domínio moradia/habitar, 8 itens no domínio rede/cuidado em comunidade, 10 itens no domínio rede social, e 5 itens no domínio renda/trabalho.

Dividimos, então, para maior facilidade no padrão de respostas do interlocutor, a escala em itens positivos, onde propomos a mensuração no que diz respeito à satisfação (1 - muito insatisfeito, 2 - insatisfeito, 3 - indiferente, 4 - satisfeito, 5 muito satisfeito) e itens negativos, que mensuram a visão do usuário acerca dos prejuízos que se apresentam em sua vida (1 prejudica muito, 2 - prejudica um pouco, 3 - indiferente, 4 - quase não prejudica, 5 - nunca prejudica).

\section{Avaliação dos Juízes Especialistas e IVC}

O IVC da escala foi de 0,85 para pertinência e 0,847 para adequabilidade, como exposto na Tabela 2. 
Tabela 2: Índice de validade de conteúdo.

\begin{tabular}{|c|c|c|c|c|}
\hline$\cdots$ & $\begin{array}{c}\text { Total de itens } \\
\text { considerado } \\
\text { pertinentes por ambos }\end{array}$ & $\begin{array}{l}\text { Total de itens } \\
\text { considerado } \\
\text { adequados por } \\
\text { ambos }\end{array}$ & $\begin{array}{c}\text { Índice de } \\
\text { Validade de } \\
\text { Conteúdo } \\
\text { Pertinência }\end{array}$ & $\begin{array}{c}\text { Índice de } \\
\text { Validade de } \\
\text { Conteúdo } \\
\text { Adequabilidade }\end{array}$ \\
\hline Concordância juiz 1 e 2 & 31 & 32 & 0,94 & 0,97 \\
\hline Concordância juiz 1 e 3 & 22 & 21 & 0,67 & 0,64 \\
\hline Concordância juiz 1 e 4 & 31 & 32 & 0,94 & 0,97 \\
\hline Concordância juiz 1 e 5 & 31 & 32 & 0,94 & 0,97 \\
\hline Concordância juiz 2 e 3 & 22 & 21 & 0,67 & 0,64 \\
\hline Concordância juiz 2 e 4 & 33 & 33 & 1,00 & 1,00 \\
\hline Concordância juiz 2 e 5 & 33 & 33 & 1,00 & 1,00 \\
\hline Concordância juiz 3 e 4 & 22 & 21 & 0,67 & 0,64 \\
\hline Concordância juiz 3 e 5 & 22 & 21 & 0,67 & 0,64 \\
\hline Concordância juiz 4 e 5 & 33 & 33 & 1,00 & 1,00 \\
\hline Médias & 28 & 27,9 & 0,85 & 0,847 \\
\hline
\end{tabular}

Fonte: Autoras.

Um item foi excluído por ter sido considerado repetido pelos juízes, e foi combinado com outro item para a versão final. As alterações que os juízes sugeriram foram relacionadas a questões de escrita e gramaticais como, por exemplo, falta de crase, erros de concordância, falta da referência à "satisfação" (que é usada para medir os itens positivos nas possibilidades de resposta) ou à palavra "prejuízo", (que é utilizada para medir os itens negativos nas possibilidades de resposta) na raiz do item , substituir siglas pela palavra por extenso (exemplo: AMA - Assistência Médica Ambulatorial), incluir questão sobre a participação do indivíduo em projetos de geração de trabalho e renda.

Para realizar as correções sugeridas pelos juízes, os autores da escala se reuniram com um especialista em mensuração e psicometria e fizeram as alterações de forma conjunta de acordo com o consenso do grupo. Após avaliações dos juízes, a escala em sua versão final possui 32 itens, pois os itens “A renda de seu trabalho atende as suas necessidades financeiras?" e "Recebe renda de outras fontes que atende às suas necessidades financeiras (auxílios, ajuda de familiares, aluguel, investimentos, benefícios sociais, e outros)?" foram combinados no novo item "Está satisfeito com sua renda (considere trabalho, auxílios, ajuda de familiares, aluguel, investimentos, benefícios sociais, e outros)?” A Tabela 3 apresenta as versões submetidas aos juízes e final dos itens. 
Tabela 3: Itens da escala de Reabilitação Psicossocial para o Usuário de SPA em sua versão avaliada pelos juízes e final. São Paulo, Brasil, 2020.

\begin{tabular}{|c|c|c|}
\hline $\begin{array}{l}\text { Item, } \\
\text { Variável/ } \\
\text { domínio }\end{array}$ & Versão autores & Versão final e revisada \\
\hline $1-\mathrm{RS}$ & $\begin{array}{l}\text { Sente que seu consumo de substâncias atrapalha a } \\
\text { sua relação com seus familiares? } *\end{array}$ & $\begin{array}{l}\text { Sente que seu consumo de substâncias } \\
\text { prejudica a sua relação com seus familiares? }\end{array}$ \\
\hline 2-RS & $\begin{array}{l}\text { Sente que a sua família reclama demais de seus } \\
\text { problemas com uso de substâncias? * }\end{array}$ & $\begin{array}{l}\text { Sente se prejudicado por reclamações de sua } \\
\text { família sobre seu uso de substâncias? }\end{array}$ \\
\hline $3-\mathrm{RS}$ & $\begin{array}{l}\text { Sente que seu consumo de substâncias prejudica a } \\
\text { sua relação com seus amigos?* }\end{array}$ & $\begin{array}{l}\text { Sente que seu consumo de substâncias } \\
\text { prejudica a sua relação com seus amigos? }\end{array}$ \\
\hline $4-\mathrm{AC}$ & $\begin{array}{l}\text { Sente que seu uso de substâncias prejudica que } \\
\text { tome suas próprias decisões? } *\end{array}$ & $\begin{array}{l}\text { Sente que seu uso de substâncias prejudica } \\
\text { que tome suas próprias decisões? }\end{array}$ \\
\hline 5-RS & $\begin{array}{l}\text { Sente que seus amigos reclamam demais de seus } \\
\text { problemas com uso de substâncias? }\end{array}$ & $\begin{array}{l}\text { Sente se prejudicado por reclamações de seus } \\
\text { amigos sobre seu uso de substâncias? }\end{array}$ \\
\hline $6-\mathrm{AC}$ & $\begin{array}{l}\text { Sente que seu uso de substâncias prejudica a sua } \\
\text { capacidade de cuidar de si mesmo? }{ }^{*}\end{array}$ & $\begin{array}{l}\text { Sente que seu uso de substâncias prejudica a } \\
\text { sua capacidade de cuidar de si mesmo? }\end{array}$ \\
\hline $7-\mathrm{AC}$ & $\begin{array}{l}\text { Não se sente bem-vindo em alguns lugares por } \\
\text { causa do uso de substâncias? * }\end{array}$ & $\begin{array}{l}\text { Se sente prejudicado ao ser mal recebido em } \\
\text { alguns lugares por causa do uso de } \\
\text { substâncias? }\end{array}$ \\
\hline $8-\mathrm{AC}$ & $\begin{array}{l}\text { Sente que seu uso de substâncias te atrapalha de } \\
\text { fazer o que quer e precisa? } *\end{array}$ & $\begin{array}{c}\text { Sente que seu uso de substâncias te atrapalha } \\
\text { de fazer o que quer e precisa? }\end{array}$ \\
\hline 9-MH & $\begin{array}{l}\text { Ficou sem ter onde morar ou teve problemas de } \\
\text { moradia por causa de seu uso de substâncias? } *\end{array}$ & $\begin{array}{c}\text { Sente que seu uso de substâncias prejudicou } \\
\text { sua situação de moradia (ficou sem ter onde } \\
\text { morar ou teve problemas)? }\end{array}$ \\
\hline $10-\mathrm{TR}$ & $\begin{array}{l}\text { Seu consumo atual de substâncias prejudica a sua } \\
\text { situação financeira? } *\end{array}$ & $\begin{array}{l}\text { Se sente prejudicado financeiramente por seu } \\
\text { consumo atual de substâncias? }\end{array}$ \\
\hline $11-\mathrm{TR}$ & $\begin{array}{l}\text { Sente que seu consumo de substâncias te impede } \\
\text { de conseguir ou te atrapalha em seu trabalho?* }\end{array}$ & $\begin{array}{c}\text { Sente que seu consumo de substâncias te } \\
\text { impede de conseguir ou te prejudica em seu } \\
\text { trabalho? }\end{array}$ \\
\hline $12-\mathrm{CC}$ & $\begin{array}{l}\text { Quando precisou do CAPS (CAPSad/CAPS } \\
\text { adulto) conseguiu acesso? (se não precisou, } \\
\text { considerar nota máxima) }\end{array}$ & $\begin{array}{c}\text { Está satisfeito com o acesso ao CAPS } \\
\text { (CAPSad/CAPS adulto)? (se não precisou, } \\
\text { considerar 5) }\end{array}$ \\
\hline $13-\mathrm{CC}$ & $\begin{array}{c}\text { Os profissionais do CAPS te visitaram em casa } \\
\text { quando precisou? (se não precisou, considerar nota } \\
\text { máxima) }\end{array}$ & $\begin{array}{c}\text { Está satisfeito com as visitas dos profissionais } \\
\text { do CAPS quando precisou? (se não precisou, } \\
\text { considerar 5) }\end{array}$ \\
\hline $14-\mathrm{CC}$ & $\begin{array}{l}\text { Quando precisou, conseguiu vaga em hospitais } \\
\text { próximos à sua casa? }\end{array}$ & $\begin{array}{l}\text { Está satisfeito com conseguir vaga em } \\
\text { hospitais próximos à sua casa quando } \\
\text { precisou? (se não precisou, considerar 5) }\end{array}$ \\
\hline $15-\mathrm{CC}$ & $\begin{array}{l}\text { Quando precisou, frequentou outros serviços de } \\
\text { saúde como UPA, AMA? }\end{array}$ & $\begin{array}{l}\text { Está satisfeito com outros serviços de saúde } \\
\text { como unidades de emergência ou pronto } \\
\text { atendimento quando precisou? (se não } \\
\text { precisou, considerar 5) }\end{array}$ \\
\hline $16-\mathrm{CC}$ & $\begin{array}{c}\text { Quando teve vontade, frequentou Centros de } \\
\text { Convivência e outros espaços coletivos da } \\
\text { comunidade (CECCO, centro cultural, espaços } \\
\text { coletivos)? }\end{array}$ & $\begin{array}{c}\text { Está satisfeito com o acesso a Centros de } \\
\text { Convivência e outros espaços coletivos da } \\
\text { comunidade (centro cultural, espaços } \\
\text { coletivos)? }\end{array}$ \\
\hline
\end{tabular}




\begin{tabular}{|c|c|c|}
\hline $17-\mathrm{CC}$ & $\begin{array}{l}\text { Você teve acesso à unidade básica de saúde (posto } \\
\text { de saúde) quando precisou? }\end{array}$ & $\begin{array}{l}\text { Está satisfeito com o acesso à unidade básica } \\
\text { de saúde (posto ou centro de saúde) quando } \\
\text { precisou? (se não precisou, considerar 5) }\end{array}$ \\
\hline $18-\mathrm{CC}$ & $\begin{array}{l}\text { Os profissionais da UBS (posto de saúde) te } \\
\text { visitaram em casa quando precisou? (se não } \\
\text { precisou, considerar nota máxima) }\end{array}$ & $\begin{array}{c}\text { Está satisfeito com as visitas dos profissionais } \\
\text { da unidade básica de saúde (posto ou centro } \\
\text { de saúde) quando precisou? (se não precisou, } \\
\text { considerar 5) }\end{array}$ \\
\hline $19-\mathrm{CC}$ & $\begin{array}{l}\text { Quando precisou, foi atendido por serviços de } \\
\text { emergência como SAMU e ambulância? }\end{array}$ & $\begin{array}{c}\text { Está satisfeito com o acesso a serviços de } \\
\text { emergência como ambulância ou Serviço de } \\
\text { Atendimento Móvel de Urgência? (se não } \\
\text { precisou, considerar 5) }\end{array}$ \\
\hline 20-RS & $\begin{array}{l}\text { Está satisfeito com a companhia de seus } \\
\text { familiares? }\end{array}$ & $\begin{array}{l}\text { Está satisfeito com a companhia de seus } \\
\text { familiares? }\end{array}$ \\
\hline 21-RS & $\begin{array}{l}\text { Sente que pode contar com seus familiares em } \\
\text { momentos difíceis? }\end{array}$ & $\begin{array}{c}\text { Está satisfeito com o quanto pode contar com } \\
\text { seus familiares em momentos difíceis? }\end{array}$ \\
\hline 22-RS & Faz atividades prazerosas com seus familiares? & $\begin{array}{c}\text { Está satisfeito com as atividades prazerosas } \\
\text { que faz com seus familiares? }\end{array}$ \\
\hline 23-RS & $\begin{array}{l}\text { Está satisfeito com a companhia de seus amigos } \\
\text { (colegas, companheiros)? }\end{array}$ & $\begin{array}{l}\text { Está satisfeito com a companhia de seus } \\
\text { amigos (colegas, companheiros)? }\end{array}$ \\
\hline 24-RS & $\begin{array}{l}\text { Sente que pode contar com seus amigos (colegas, } \\
\text { companheiros) em momentos difíceis? }\end{array}$ & $\begin{array}{c}\text { Está satisfeito com o quanto pode contar com } \\
\text { seus amigos (colegas, companheiros) em } \\
\text { momentos difíceis? }\end{array}$ \\
\hline $25-\mathrm{RS}$ & $\begin{array}{l}\text { Faz atividades prazerosas com seus amigos } \\
\text { (colegas, companheiros)? }\end{array}$ & $\begin{array}{l}\text { Está satisfeito com as atividades prazerosas } \\
\text { que faz com seus amigos (colegas, } \\
\text { companheiros)? }\end{array}$ \\
\hline 26-MH & Está satisfeito com o local onde mora? & $\begin{array}{c}\text { Sente que seu uso de substâncias prejudicou } \\
\text { sua situação de moradia (ficou sem ter onde } \\
\text { morar ou teve problemas)? }\end{array}$ \\
\hline N/A-TR & $\begin{array}{l}\text { A renda de seu trabalho atende as suas } \\
\text { necessidades financeiras? }\end{array}$ & (Item excluído, combinado aoitem 26 abaixo) \\
\hline 27-TR & $\begin{array}{l}\text { Recebe renda de outras fontes que atende as suas } \\
\text { necessidades financeiras (auxílios, ajuda de } \\
\text { familiares, aluguel, investimentos, benefícios } \\
\text { sociais, e outros)? }\end{array}$ & $\begin{array}{l}\text { Está satisfeito com sua renda (considere } \\
\text { trabalho, auxílios, ajuda de familiares, aluguel, } \\
\text { investimentos, benefícios sociais, e outros)? }\end{array}$ \\
\hline 28-TR & Tem um trabalho que te traz realização pessoal? & $\begin{array}{l}\text { Está satisfeito com seu emprego ou sua } \\
\text { situação de trabalho? }\end{array}$ \\
\hline 29-MH & Se sente seguro no local onde mora? & $\begin{array}{l}\text { Está satisfeito com a segurança no local onde } \\
\text { mora? }\end{array}$ \\
\hline 30-MH & $\begin{array}{l}\text { Sente que tem liberdade para fazer o que tem } \\
\text { vontade no local onde mora? }\end{array}$ & $\begin{array}{l}\text { Está satisfeito com a liberdade para fazer o } \\
\text { que tem vontade no local onde mora? }\end{array}$ \\
\hline 31-MH & $\begin{array}{c}\text { Caso esteja em situação de rua, está satisfeito com } \\
\text { esta condição? (caso não esteja em situação de rua, } \\
\text { considerar nota máxima) }\end{array}$ & $\begin{array}{c}\text { Caso esteja em situação de rua, está satisfeito } \\
\text { com esta condição? (caso não esteja em } \\
\text { situação de rua, considerar 5) }\end{array}$ \\
\hline 32-MH & Sente confortável no local onde mora? & $\begin{array}{c}\text { Está satisfeito com o conforto do local onde } \\
\text { mora? }\end{array}$ \\
\hline
\end{tabular}

Legendas: * Itens negativos. Domínios: RS - Rede Social, AC - Autonomia/Cidadania, MH - Moradia/Habitar, CC - Cuidado na Comunidade, TR - Trabalho e Renda. N/A - Não se aplica. Fonte: Autores. 


\section{Discussão}

Mediante leitura e análise dos dados encontrados por meio da revisão da literatura nas bases de dados, observamos a importância da RP para o indivíduo, sua família e também para os profissionais dos equipamentos de saúde que trabalham com a assistência à população que faz uso abusivo de SPA.

A necessidade de estudo e pesquisa nesse campo da Saúde Mental é fundamental para elevar o nível de conhecimento e instrumentalização de ambas as partes, usuários e profissionais, uma vez que seu conceito e princípios parecem ainda não serem muito claros, como demonstra o estudo de Pinho et al., (2013) que buscou as impressões de 11 profissionais de saúde de um Centro de Referência para o tratamento dos problemas relacionados ao uso de SPA acerca da RP, compreendendo que os profissionais de saúde ainda estão muito atrelados ao modelo tradicional de "normalidade social", ou seja, o habitat como propriedade, a rede social na busca ativa de familiares e atividades externas, deixando escapar a rede social mais ampla e o trabalho voltado para oficinas e parcerias, utilizado como uma ferramenta que visa apenas eliminar a ociosidade e proporcionar entretenimento.

Ainda, quanto à imprecisão do conceito de RP voltada aos usuários de SPA uma revisão de literatura evidenciou que os termos reabilitação psicossocial, reinserção social e inclusão social são frequentemente utilizados como sinônimos nos trabalhos acadêmicos, demonstrando a necessidade de estudos nesta área (Sanches \& Vecchia, 2018).

Sob a ótica dos consumidores de SPA o mundo é dicotômico, o mundo sem as drogas e o mundo das drogas, que por sua vez é o mundo das pessoas sem valor, sem conhecimento e sem cidadania. Quando questionados, os usuários de um CAPS AD se consideram fora da sociedade e com sentimentos constantes de humilhação, inferioridade e submissão. Ainda, é desconhecido por parte dos usuários, o princípio que norteia o tratamento oferecido nos CAPS, identificado em falas como "tratamento total" e "internação". O que demonstra em conjunto o desconhecimento da RP como oportunidade para novos projetos de vida (Sena et al., 2017).

Os artigos selecionados mostraram, também, maior prevalência de problemas decorrentes do uso de SPA entre os jovens adultos (18 a 25 anos), com pico aos 21 anos, o que indica a idade adulta emergente como ponto crucial para desenvolvimento de ações problemas graves e duradouros do uso de SPA e demonstra a necessidade de mais estudos para abordagens específicas para esta faixa etária (Sheidow et al., 2012; Sena et al., 2017). Entretanto, os dados apresentados surgem de uma revisão integrativa que avaliou os jovens americanos, que contam com políticas públicas divergentes ao Brasil, onde citam que o pico de consumo aos 21 anos é coerente com a legislação local, que só permite a compra e consumo de bebidas alcoólicas nesta mesma idade (Sheidow et al., 2012). A escassez de pesquisa nesta área no Brasil limita inferirmos dados como estes, apesar da permissividade por idade ser relativamente próxima (18 anos para os brasileiros e 21 para os americanos) esbarramos em restrições não apenas legislativas como, também, culturais.

Sabe-se que o consumo de álcool e outras drogas por jovens está atrelado entre outros fatores a quadros de transtorno mental, classificando tal ação como uma comorbidade psiquiátrica. Com base nesta afirmação, a rede de assistência apoiada por recursos extra hospitalares, foca sua ação na atenção psicossocial, e assim, articulada com outros setores do serviço de saúde, ressaltam a RP e a reinserção social dos indivíduos que apresentem problemas oriundos do uso de álcool e outras drogas (Pinho et al., 2008; Sheidow et al., 2012).

Ainda sobre os jovens, Green et al. (2012), procuraram orientações para engajar os mais novos que estão em tratamento pelo uso de SPA, por intermédio dos usuários mais antigos. O estudo resultou em importantes recomendações, que são preconizadas pela RP, como a abordagem pessoal e não diagnóstica, adequar o tratamento a linguagem e cultura do jovem, sendo empático, acolhedor, autêntico e, principalmente, estimulando a autonomia do indivíduo em um ambiente seguro, prevenindo o isolamento social. Assim, as sugestões dos usuários mais antigos falam, em outras palavras, dos eixos: morar/habitar e da rede social. 
Além dos temas legislativos e culturais levantadas, encontramos, também, um fenômeno de ordem estrutural e conjuntural, que é o desemprego. Estudo que buscou avaliar a satisfação de 25 usuários de um CAPS AD no interior de São Paulo e, apesar de conceber que grande parte deles estavam satisfeitos com a competência e a compreensão da equipe, identificou que o desemprego é significativo para aqueles que fazem consumo de álcool e drogas, uma vez que são estigmatizados, marginalizados e excluídos socialmente (Barbosa et al., 2015).

O estigma que circunda os sujeitos que consomem álcool e drogas é uma das grandes barreiras para as atividades relacionadas ao trabalho e a geração de renda, além de também afetar a relação do usuário com sua família e com a sociedade em geral (Sanches \& Vecchia, 2018). Notoriamente, vínculos afetivos com pessoas importantes para o usuário devem ser estimulados e representam parte do que é a RP, porém, não se pode perder de vista que este é apenas um de seus eixos, que fazem parte de um todo complexo.

Três periódicos abordaram a importância do eixo "rede social" para os usuários de SPA e para a RP. Pinho et al., (2008) descrevem, em revisão bibliográfica, os termos "apoio/suporte social" como fundamentais no tratamento de problemas decorrentes do consumo de álcool e drogas, entretanto, tais termos não são relacionados, na literatura internacional, com a RP. Em um dos estudos o autor discute, similarmente, a elevação da qualidade e eficácia do tratamento quando é realizado de modo compartilhado entre profissionais e familiares, que também devem receber suporte terapêtico, uma vez que foram marcados com traumas da vivência com os usuários de SPA (Alvarez et al., 2012). Em outra pesquisa, os autores também citam as intervenções multidisciplinares com abordagem cognitiva comportamental e o envolvimento de pessoas significativas ao usuário como os melhores resultados nos indicadores de abstinência e prevenção de recaídas (Lefio et al., 2013).

Na perspectiva do trabalho em equipe interdisciplinar, o planejamento das atividades do projeto terapêutico singular devem se caracterizar por intervenções fundamentadas na reabilitação psicossocial, sobretudo nos três eixos: rede social, trabalho e moradia (Zotesso et al., 2019). Nessa direção, é possível verificar que há muito trabalho e empenho de profissionais envolvidos nas estratégias de RP, mas ainda há um déficit na esfera de reinserção desses indivíduos de uma forma ativa na sociedade, ou seja, de maneira a reduzir o estigma de incapacidade que esses sujeitos carregam, ficando as ações ainda muito interiorizadas nos serviços.

A RP como processo, não tem um fim definido, mas somando-se todos esses pontos relacionados ao acesso material, habitacional e laboral, pode-se alcançar o objetivo que integra a volta destes indivíduos para a sociedade, ou seja, a reinserção social completa (Goldberg, 2001).

Por fim, o processo reabilitatório e o tratamento dos usuários de SPA é um fenômeno complexo, e por esse motivo, a reabilitação destes indivíduos em todos os seus domínios, requer redes de assistência apropriadas às variadas necessidades desse segmento da população como: o cuidado em saúde, benefícios sociais, esporte, lazer, cultura, moradia, trabalho, educação entre outros.

O Índice de Validade de Conteúdo da escala é considerado válido pela literatura especializada tanto no que tange à para pertinência (IVC $=0,85)$ dos itens e quanto à adequabilidade à população alvo (IVC = 0,847) (Polit et al., 1995; Alexandre \& Coluci, 2011).

$\mathrm{Na}$ análise pelos juízes, foi possível assimilar melhorias aos itens que farão com que a escala seja melhor compreendida pela população alvo. Os juízes contribuíram não somente para uma melhor configuração dos itens, mas também com ideias de como agrupá-los de forma que os indivíduos a compreendam melhor e possam respondê-la mais facilmente, apresentando primeiro as questões positivas, onde questiona-se sobre a satisfação que o indivíduo percebe em sua vida, e, após, apresentando as questões negativas, onde abordamos prejuízos que ele detecta em sua vida. Estudos anteriores utilizaram técnica semelhante para incorporar as sugestões dos juízes (Alexandre \& Coluci, 2011; Claro et al., 2012). 
Propomos que o escore para a escala seja calculado pela soma simples dos valores atribuídos a cada resposta, resultando em um escore mínimo de 32 (indivíduo com mais demandas para a garantia de sua RP) e máximo de 160 pontos (indivíduo com menos demandas para garantia de sua RP).

\section{Considerações Finais}

A literatura sobre o tema ainda é escassa e recente, contudo, por meio dos dados encontrados pela revisão integrativa, foi possível propor uma escala que mensura aspectos relacionados à RP.

A escala nos cinco domínios, trabalho/renda, moradia, rede social, autonomia/cidadania e cuidado na comunidade, permite identificar áreas de vulnerabilidade do indivíduo, contribuindo de forma objetiva com a avaliação, planejamento e construção do Projeto Terapêutico Singular dos usuários. Contribui também na avaliação dos serviços da Rede de Atenção Psicossocial, com base na promoção da RP.

A "Escala de Reabilitação Psicossocial para Usuários de Substâncias Psicoativas" possui validade de conteúdo e a análise de outras propriedades psicométricas contribuirá para a construção de evidências. Sugere-se para trabalhos futuros a validação da escala com a população que realiza tratamento nos serviços comunitários de saúde mental para o uso abusivo de substâncias psicoativas.

\section{Referências}

Alexandre N. M. C., \& Coluci M. Z. O. (2011). Validade de conteúdo nos processos de construção e adaptação de instrumentos de medidas. Cien Saude Colet, 16(7), 3061-8.

Alvarez S. Q., Gomes G. C., Oliveira A. M. N., \& Xavier D. M. (2012). Grupo de apoio/suporte como estratégia de cuidado: importância para familiares de usuários de drogas. Rev Gaúcha de Enferm, 102-8. http://dx.doi.org/10.1590/s1983-14472012000200015.

Barbosa G. C., Oliveira M. A. F., Moreno V., Padovani C. R., Claro H. G., \& Pinho P. H. (2015). Satisfação de usuários num Centro de Atenção Psicossocial em álcool e outras drogas. Rev Port Enferm Saúde Mental, (14), 31-7.

Bellack A. S., \& Mueser K. T. (1993). Psychosocial Treatment for Schizophrenia. Schizophr Bull. Schizophrenia Bulletin, (19), 317-36. http://dx.doi.org/10.1093/schbul/19.2.317.

Bergman H. C., \& Harris M. (1985). Substance abuse among young adult chronic patients. Psychosoc Rehabil J., 49-54. http://dx.doi.org/10.1037/h0099162.

Blondell R. D., Frydrych L. M., Jaanimagi U., Ashrafioun L., Homish G. G., Foschio E. M., \& Bashaw, H. L. (2011). A randomized trial of two behavioral interventions to improve outcomes following inpatient detoxification for alcohol dependence. J Addict Dis, 30(2), $136-48$.

Bohn M. J. (1993). Alcoholism. Psychiatr Clin North Am, 16(4), 679-92.

Brito A. A. C., Brito A. A. C., Silva D. S., e Azevedo D. M. (2012). Formação do acadêmico de enfermagem: vivência na atenção a usuários de drogas psicoativas. Esc Anna Nery, 395-400. http://dx.doi.org/10.1590/s1414-81452012000200026.

Braucht G. N., Kirby M. W., \& Berry G. J. (1978). Psychosocial correlates of empirical types of multiple drug abusers. J Consult Clin Psychol, 46(6), 146375.

Claro H. G., Oliveira M. A. F., Paglione H. B., Pinho P. H., Pereira M. O., \& Vargas D. (2012). Tradução e adaptação cultural do global appraisal of individual needs - initial. Rev Esc Enferm USP, 1148-55. http://dx.doi.org/10.1590/s0080-62342012000500016.

Claro H. G., Oliveira M. A. F., Almeida, Mastrocolla M., Vargas D., \& Paglione H. B. (2011). Adaptação cultural de instrumentos de coleta de dados para mensuração em álcool e drogas. SMAD. Revista eletrônica saúde mental álcool e drogas, 7(2), 71-77. http://pepsic.bvsalud.org/scielo.php?script=sci_arttext\&pid=S1806-69762011000200004\&lng=pt\&tlng=pt.

Davison G. C. (2000). Stepped care: doing more with less? J Consult Clin Psychol, 68(4), 580-5.

Dell'Osso B., Vismara M., Dobrea C., Cremaschi L., Grancini B., Arici C., Benatti B., Buoli M., Kettler T. A., \& Altamura A. C. (2018). Clinical characterization of Italian suicide attempters with bipolar disorder. Espectro CNS, 23(4), 271-7.

Fals-Stewart W., \& O'Farrell T. J. (2003). Behavioral family counseling and naltrexone for male opioid-dependent patients. J Consult Clin Psychol, 71(3), $432-42$.

Ferrari A. L. (2009). Adaptação Transcultural do Questionário Cultural Study of Musculo-Skeletal and Other Symptoms and Assocated Disability - CUPID Questionnaire. Dissertação, Escola de Enfermagem - Universidade de São Paulo, São Paulo, SP, Brasil. 
Gavioli E. C. (2017). Topiramate reduces basal anxiety and relieves ethanol withdrawal-induced anxious behaviors in male rats. Exp Clin Psychopharmacol, 25(2), 105-13.

George F. R., \& Ritz M. C. (1993). A psychopharmacology of motivation and reward related to substance abuse treatment. Exp Clin Psychopharmacol, 7-26. Available from: http://dx.doi.org/10.1037/1064-1297.1.1-4.7.

Goldberg, J. (2001). Reabilitação psicossocial no Brasil. (Reabilitação como processo: O centro de atenção psicossocial - CAPS, pp. 33-47). São Paulo: Hucitec.

Green C. A., Wisdom J. P., Wolfe L., \& Firemark A. (2012). Engaging youths with serious mental illnesses in treatment: STARS study consumer recommendations. Psychiatr Rehabil J, 35(5), 360-8.

Hyde A. P., \& Goldman C. R. (1993). Common family issues that interfere with the treatment and rehabilitation of people with schizophrenia. Psychosoc Rehabil J, p. 63-74. Available from: http://dx.doi.org/10.1037/h009565.

Judd P. H., Thomas N., Schwartz T., Outcalt A., \& Hough R. (2003). A dual diagnosis demonstration project: treatment outcomes and cost analysis. $J$ Psychoactive Drugs, 35(1), 181-92.

Junqueira-Ayres D. D., Asth L., Ayres A. S. F. S. J., Lobão-Soares B., Soares-Rachetti V. P., \& Gavioli E.C. (2017). Topiramate reduces basal anxiety and relieves ethanol withdrawal-induced anxious behaviors in male rats. Exp Clin Psychopharmacol, 25(2), 105-13.

Lazar C. M., Black A. C., McMahon T. J., Rosenheck R. A., Ries R., Ames D., \& Rosen M. I. (2016). All-data approach to assessing financial capability in people with psychiatric disabilities. Psychol Assess, 28(4), 362-71.

Lefio L. Á., Villarroel S. R., Rebolledo C., Zamorano P., \& Rivas K. (2013). Intervenciones eficaces en consumo problemático de alcohol y otras drogas. Rev Panam Salud Publica, (34), 257-66.

Lucchese R., Silva P. C. D., Denardi T. C., Felipe R. L., Vera I., Castro P. A., Bueno A. A., \& Fernandes I. L. (2017). Common mental disorder among alcohol and drug abusers: a cross-sectional study. Texto \& Contexto Enferm. Available from: http://dx.doi.org/10.1590/0104-07072017004480015.

Lei n. 10.216, de 06 de abril de 2001 (2001). Dispõe sobre a proteção e os direitos das pessoas portadoras de transtornos mentais e redireciona o modelo assistencial em Saúde Mental. Brasília, DF: Presidência da República.

Liposck D. B., Rosa Neto F., \& Savall A. C. (2007). Validação do conteúdo do instrumento de avaliação postural - IAP. Revista Digital Buenos Aires, 12(109).

Maisto S. A., Martin C. S., Pollock N. K., Cornelius J. R., \& Chung T. A. (2002). Nonproblem drinking outcomes in adolescents treated for alcohol use disorders. Exp Clin Psychopharmacol, 10(3), 324-31.

McCoy M. L., Devitt T., Clay R., Davis K. E., Dincin J., Pavick D., \& O’Neill S. (2003). Gaining insight: who benefits from residential, integrated treatment for people with dual diagnosis? Psychiatr Rehabil J, 27(2), 140-50.

McKay J. R., Drapkin M. L., Van Horn D. H. A., Lynch K. G., Oslin D. W., DePhilippis D., Ivey M., \& Cacciola J. S. (2015). Effect of patient choice in an adaptive sequential randomization trial of treatment for alcohol and cocaine dependence. J Consult Clin Psychol, 1021-32. http://dx.doi.org/10.1 037/a0039534.

Ministério da Saúde. A Política do Ministério da Saúde para a Atenção Integral a Usuários de Álcool e outras Drogas. (2004). Brasília: Ministério da Saúde.

Otto-Salaj L. L., Kelly J. A., \& Stevenson L. Y. (1998). Implementing cognitive-behavioral AIDS/HIV risk reduction group interventions in community mental health settings that serve people with serious mental illness. Psychiatr Rehabili J, 394-404. Available from: http://dx.doi.org/10.1037/h0095283.

Pinho P. H., Oliveira M. A. F., Vargas D., Almeida M. M., Machado A. L., Silva A. L. A., Colvero L. A., \& Barros S. (2009). Reabilitação psicossocial dos usuários de álcool e outras drogas: a concepção de profissionais de saúde. Rev Esc Enferm USP, 1261-6. http://dx.doi.org/10.1590/s008062342009000600020 .

Pinho P. H., Oliveira M. A., \& Almeida M. M. (2008). A reabilitação psicossocial na atenção aos transtornos associados ao consumo de álcool e outras drogas: uma estratégia possível? Rev Psiquiatr Clín, 35(1), 82-8.

Pinho P. H., Oliveira M. F., Claro H. G., Pereira M. O., \& Almeida M. M. (2013). A concepção dos profissionais de saúde acerca da reabilitação psicossocial nos eixos: morar, rede social e trabalho dos usuários de substâncias psicoativas. Rev Port Enferm saúde mental, (9), 29-35.

Polit D. F., Beck C. T., \& Hungler B. P. (1995). Fundamentos de pesquisa em enfermagem, (3). Porto Alegre: Artes Médicas.

Portaria n. 336/GM, de 19 de fevereiro de 2002 (2002). Dispõe sobre os Centros de Atenção Psicossocial. Ministério da Saúde. Brasília, DF. Available from: http://portal.saude.gov.br/portal/arquivos/pdf/Portaria

Sanches L. R., \& Vecchia M. D. (2018). Reabilitação psicossocial e reinserção social de usuários de drogas: revisão da literatura. Psicologia \& Sociedade, 30, e178335. https://doi.org/10.1590/1807-0310/2018v30178335.

Sanches L. R., \& Vecchia M. D. (2020). Reabilitação psicossocial e inclusão social de pessoas com problemas decorrentes do uso de álcool e outras drogas: impasses e desafios. Interface (Botucatu); 24: e200239. https://doi.org/10.1590/interface.200239

Saraceno B. (1999). Libertando identidades: da reabilitação psicossocial à cidadania possível. Te Cora.

Secades-Villa R., García-Rodríguez O., García-Fernández G., Sánchez-Hervás E., Fernandez-Hermida JR., \& Higgins S. T. (2011). Community reinforcement approach plus vouchers among cocaine-dependent outpatients: twelve-month outcomes. Psychol Addict Behav, 25(1), 174-9. 
Research, Society and Development, v. 10, n. 7, e17710716418, 2021

(CC BY 4.0) | ISSN 2525-3409 | DOI: http://dx.doi.org/10.33448/rsd-v10i7.16418

Sena E. L. S., Soares C. J., Ribeiro B. S., Santos P. H. S., Carmo E. A., \& Carvalho P. A. L. (2017). Reabilitação psicossocial segundo a perspectiva de consumidores de drogas Psychosocial rehabilitation according to drug consumers' perspective. Revista de Pesquisa: Cuidado é Fundamental Online, 520. http://dx.doi.org/10.9789/2175-5361.2017.v9i2.520-525.

Sheidow A. J, McCart M., Zajac K., \& Davis M. (2012). Prevalence and impact of substance use among emerging adults with serious mental health conditions. Psychiatr Rehabil J, 35(3), 235-43.

Soares C. B., Hoga L. A. K., Peduzzi M., Sangaleti C., Yonekura T., \& Silva D. R. A. D. (2014). Revisão integrativa: conceitos e métodos utilizados na enfermagem. Rev Esc Enferm USP, 48(2), 335-45.

Sordi L. D., Sordi L., Bigatto K., Santos S., \& Machado A. L. (2015). Comorbidades em usuários de um serviço de saúde mental. Portuguese Journal of Mental Health Nursing. Available from: http://dx.doi.org/10.19131/jpmhn.0014.

Stein L. A. R, Clair M., Rossi J.S., Martin R. A., Cancilliere M. K., e Clarke J. G. (2015). Gender, ethnicity and race in incarcerated and detained youth: services and policy implications for girls. Psychiatr Rehabil J, 38(1), 65-73.

UNODC. World Drug Report 2014. Naciones Unidas Nueva York. (2014). http://www.unodc.org/documents/wdr2014/World_Drug_Report_2014_web.pdf.

Waltz C., \& Bausell R.B. (1981). Nursing Research: Design, Statistics and Computer Analysis. FA Davis Company, 362.

Waltz C. F., Strickland O., \& Lenz E. R. (1991). Measurement in Nursing Research. Philadelphia: FA Davis Company, (3), 533.

Wandekoken K. D., Quintanilha B. C., \& Dalbello-Araujo M. (2015). Biopolítica na Assistência aos Usuários de Álcool e Outras Drogas. Revista Subjetividade, 389-97. http://dx.doi.org/10.5020/23590777.15.3.389-397.

White K. K., \& White D. E. (1989). Dual mental health and substance use problems: A model of four subtypes. Psychosoc Rehabil J, 93-8. http://dx.doi.org/10.1037/h0099510.

Zotesso M. C., Marques L. O., \& Paiva S. M. A. (2019). Centro de Atenção Psicossocial de álcool e outras drogas: práticas desenvolvidas pelos profissionais de saúde. Revista Psicologia, Diversidade e Saúde, 8(1), 8-16. 10.17267/2317-3394rpds.v8i1.2220. 